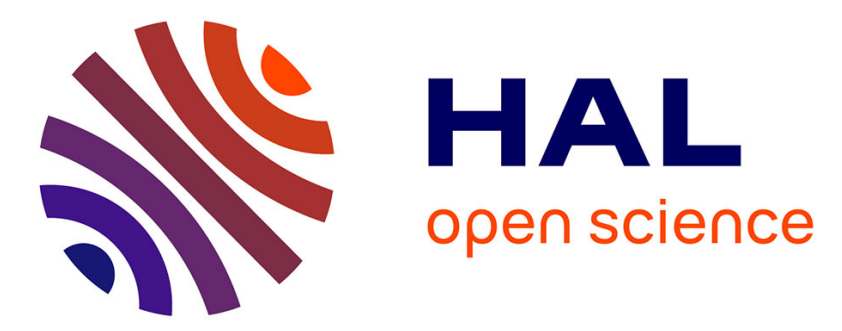

\title{
GLOBAL NON-NEGATIVE APPROXIMATE CONTROLLABILITY OF PARABOLIC EQUATIONS WITH SINGULAR POTENTIALS
}

Judith Vancostenoble

\section{> To cite this version:}

Judith Vancostenoble. GLOBAL NON-NEGATIVE APPROXIMATE CONTROLLABILITY OF PARABOLIC EQUATIONS WITH SINGULAR POTENTIALS. 2018. hal-01895349

\author{
HAL Id: hal-01895349 \\ https://hal.science/hal-01895349
}

Preprint submitted on 15 Oct 2018

HAL is a multi-disciplinary open access archive for the deposit and dissemination of scientific research documents, whether they are published or not. The documents may come from teaching and research institutions in France or abroad, or from public or private research centers.
L'archive ouverte pluridisciplinaire HAL, est destinée au dépôt et à la diffusion de documents scientifiques de niveau recherche, publiés ou non, émanant des établissements d'enseignement et de recherche français ou étrangers, des laboratoires publics ou privés. 


\title{
GLOBAL NON-NEGATIVE APPROXIMATE CONTROLLABILITY OF PARABOLIC EQUATIONS WITH SINGULAR POTENTIALS
}

\author{
J. VANCOSTENOBLE
}

\begin{abstract}
In this work, we consider the linear $1-d$ heat equation with some singular potential (typically the so-called inverse square potential). We investigate the global approximate controllability via a multiplicative (or bilinear) control. Provided that the singular potential is not super-critical, we prove that any non-zero and non-negative initial state in $L^{2}$ can be steered into any neighborhood of any non-negative target in $L^{2}$ using some static bilinear control in $L^{\infty}$. Besides the corresponding solution remains non-negative at all times.
\end{abstract}

\section{INTRODUCTION AND MAIN RESULTS}

I.1. Introduction. In this paper, we analyze controllability properties for parabolic equations with singular potential. Typically, we consider the following linear $1-D$ heat equation with an inverse square potential (that arises for example in the context of combustion theory or quantum mechanics):

$$
\begin{cases}u_{t}-u_{x x}-\frac{\mu}{x^{2}} u=0 & x \in(0,1), t>0, \\ u(0, t)=0=u(1, t) & t>0, \\ u(x, 0)=u_{0}(x) & x \in(0,1),\end{cases}
$$

where $u_{0} \in L^{2}(0,1)$ and $\mu$ is a real parameter. We concentrate on the above typical problem to simplify the presentation. However notice that this work covers more general cases that are mentionned later in section I.4.

Since the pioneering works by Baras and Goldstein $[2,3]$, we know that inverse square potentials generate interesting phenomena. In particular, existence/blow-up of positive solutions is determined by the value of $\mu$ with respect to the constant $\mu^{\star}=1 / 4$ appearing in the Hardy inequality $[18,25]$ :

$$
\forall z \in H_{0}^{1}(0,1), \quad \frac{1}{4} \int_{0}^{1} \frac{z^{2}}{x^{2}} d x \leq \int_{0}^{1} z_{x}^{2} d x .
$$

When $\mu<1 / 4$, the operator $z \mapsto-z_{x x}-\mu x^{-2} z$ generates a coercive quadratic form in $H_{0}^{1}(0,1)$. This allows showing the well-posedness in the classical variational setting of the linear heat equation with smooth coefficients, that is: for any $u_{0} \in L^{2}(0,1)$, there exists a unique solution $u \in \mathcal{C}\left(\left[0,+\infty\left[; L^{2}(0,1)\right) \cap\right.\right.$ $L^{2}\left(0,+\infty ; H_{0}^{1}(0,1)\right)$.

For the critical value $\mu=1 / 4$, the space $H_{0}^{1}(0,1)$ has to be slightly enlarged as shown in [30] but a similar result of well-posedness occurs. (See section II for details).

Finally, when $\mu>1 / 4$, the problem is ill-posed (due to possible instantaneous blow-up) as proved in [2].

Recently, the null controllability properties of (I. 1) began to be studied. For any $\mu \leq 1 / 4$, it has been proved in [29] that such equations can be controlled (in

Date: October 11, 2018 
any time $T>0$ ) by a locally distributed control: $\forall \mu \leq 1 / 4, \forall u_{0} \in L^{2}(0,1), \forall T>0$, $\forall 0 \leq a<b \leq 1$, there exists $h \in L^{2}((0,1) \times(0, T))$ such that the solution of

$$
\begin{cases}u_{t}-u_{x x}-\frac{\mu}{x^{2}} u=h(x, t) \chi_{(a, b)}(x) & x \in(0,1), t \in(0, T), \\ u(0, t)=0=u(1, t) & t \in(0, T), \\ u(x, 0)=u_{0}(x) & x \in(0,1),\end{cases}
$$

satisfies $u(\cdot, T) \equiv 0$. On the contrary, when $\mu>\mu_{\star}=1 / 4$, the property fails as shown in [12].

After these first results, several other works followed extending them in various situations. See for instance $[4,10,24,27,28]$. In (I. 3), $h \chi_{[a, b)}$ represents a locally distributed control that enters the model as an additive term describing the effect of some external force or source on the process at hand. However this is not always realistic to act on the system in such a way.

In the present work, we are insterested in studying the effect of other kind of controls on problem (I. 1). In the spirit of the works by Khapalov [19, 20, 21, 22], we aim to consider a multiplicative (also called bilinear) control. This means that the control enters now as a multiplicative coefficient in the equation (see section I.2). The advantages of such controls mainly rely on the fact that, instead of being some external action on the system, they may represent changes of parameters of the considered process. We refer to [22, chap. 1] for a list of situations for which additive controls do not seem realistic whereas multiplicative ones provide a precious alternative. Moreover, multiplicative controls also allow to steer nonnegative initial states to non-negative targets preserving the non-negativity of the solutions during the process. Even though this last property is naturally expected in many concrete situations, this was not guaranted when dealing with additive controls!

Let us finally mention several other contributions to multiplicative controls of parabolic pde's: we refer for instance the reader to $[5,6,7,8,14,26]$ and the references therein.

I.2. Description of the multiplicative control problem. Let $T>0$ and let us consider the following Dirichlet boundary problem:

$$
\begin{cases}u_{t}-u_{x x}-\frac{\mu}{x^{2}} u=\alpha(x, t) u & (x, t) \in Q_{T}:=(0,1) \times(0, T), \\ u(0, t)=0=u(1, t) & t \in(0, T) \\ u(x, 0)=u_{0}(x) & x \in(0,1)\end{cases}
$$

Here $\alpha \in L^{\infty}\left(Q_{T}\right)$ is a control function of multiplicative/bilinear form. Our goal is to study the global approximate controllability properties of system (I. 4). So following Khapalov (see [22, chap. 2, def. 2.1]), we use the following notion:

Definition I.1. System (I. 4) is non-negatively globally approximately controllable in $L^{2}(0,1)$ if, for any $\varepsilon>0$ and for every non-negative $u_{0}, u_{d} \in L^{2}(0,1)$ with $u_{0} \neq \equiv$, there exist some $T=T\left(\varepsilon, u_{0}, u_{d}\right)$ and some bilinear control $\alpha(x, t) \in L^{\infty}\left(Q_{T}\right)$ such that the corresponding solution of (I. 4) satisfies

$$
\left\|u(\cdot, T)-u_{d}\right\|_{L^{2}(0,1)} \leq \varepsilon
$$

Besides, we say that the bilinear control is static if $\alpha=\alpha(x) \in L^{\infty}(0,1)$.

I.3. Main result. Now we are ready to state our main result concerning the case of the inverse square potential (proved later in section III):

Theorem I.1. Assume that $\mu \leq 1 / 4$. Then system (I. 4) is non-negatively globally approximately controllable in $L^{2}(0,1)$ by means of static controls $\alpha=\alpha(x)$ in 
$L^{\infty}(0,1)$. Moreover, the corresponding solution to (I. 4) remains non-negative at all times.

\section{I.4. Other results.}

I.4.a. Larger class of data. With no change in the proof of Theorem I.1, one can actually state a result that concerns a larger class of data (see the proof in section IV):

Theorem I.2. For any $u_{0}, u_{d} \in L^{2}(0,1)$ such that

$$
\left\langle u_{0}, u_{d}\right\rangle_{L^{2}(0,1)}>0 \text { and } u_{d} \geq 0,
$$

for avery $\varepsilon>0$, there exist some $T=T\left(\varepsilon, u_{0}, u_{d}\right)$ and some static bilinear control $\alpha=\alpha(x)$ in $L^{\infty}(0,1)$ such that the corresponding solution of (I. 4) satisfies

$$
\left\|u(\cdot, T)-u_{d}\right\|_{L^{2}(0,1)} \leq \varepsilon .
$$

I.4.b. General form of singular potential. We considered previously the typical case of the inverse square potential $V(x)=\mu / x^{2}$ with $\mu \leq \mu_{\star}=1 / 4$. Now we turn to more general singular potentials. Let $V$ be a locally integrable function defined on $(0,1)$ and assume that

$$
0 \leq V(x) \leq \frac{\mu}{x^{2}} \quad \text { with some } \quad \mu \leq \mu_{\star}=\frac{1}{4} .
$$

For $T>0$, we now consider the following Dirichlet boundary problem:

$$
\begin{cases}u_{t}-u_{x x}-V(x) u=\alpha(x, t) u & (x, t) \in Q_{T}:=(0,1) \times(0, T), \\ u(0, t)=0=u(1, t) & t \in(0, T), \\ u(x, 0)=u_{0}(x) & x \in(0,1) .\end{cases}
$$

And we prove (see section IV):

Theorem I.3. Assume that $V(x)$ satisfies (I. 5). Then system (I. 6) is nonnegatively globally approximately controllable in $L^{2}(0,1)$ by means of static controls $\alpha=\alpha(x)$ in $L^{\infty}(0,1)$. Moreover, the corresponding solution to (I. 6) remains non-negative at all times.

\section{I.5. Perpectives.}

I.5.a. Degenerate/singular heat equation. This present work complements $[5,6,14]$ where the case of the heat equation with some degenerate diffusion coefficient

$$
u_{t}-\left(x^{\alpha} u_{x}\right)_{x}=0, \quad x \in(0,1),
$$

was investigated. Here $\alpha \geq 0$ represents the order of degeneracy of the diffusion coefficient that may vanish at $x=0$. Associated to suitable boundary conditions, this problem is well-posed. In the first studies of its controllability properties, it has been proved that (I. 7) is controllable via additive control if an only if $\alpha<2$, see [9]. In [5, 6, 14], the authors prove that, still asuming $\alpha<2$, it can also be controlled via multiplicative controls.

Let us now consider the following degenerate/singular equation:

$$
u_{t}-\left(x^{\alpha} u_{x}\right)_{x}-\frac{\mu}{x^{\beta}} u=0, \quad x \in(0,1) .
$$

It has been proved in [27] that, provided that the parameters $\alpha, \mu, \beta$ satisfies some precise (and optimal) conditions, problem (I. 8) is controllable via additive controls. (See also $[16,17]$ to various extensions). We expect that it should also be controllable via multiplicative controls under the same conditions on the parameters. 
I.5.b. Semilinear heat equation with singular potential. Another perspective is the study of the null controllability by multiplicative control of semilinear perturbations of the present singular heat equation. In [20], Khapalov studied the case of the classical heat equation with a semilinear term whereas the case of some degenerate heat equation has been studied by Floridia in [14]. We expect that it would be possible to get similar results as in $[20,14]$ in the case of the heat equation with a singular potential.

I.5.c. Nonnegative controllability in small time. In the present work, following the strategy introduced by Khapalov in [20], we got a result of controllability in large time. In the case of the classical heat equation, Khapalov also developped a second approach ("a qualitative approach" presented in [21]) that allows him to get a result of controllability in small time. An open and interesting question would be to obtain a similar result of controllability in small time in the case of the heat equation with a singular potential. For now, the question remains open. The proofs in [21] are strongly based on specific properties of the classical heat operator (regularity of the solutions) which are no more true when the operator is perturbed by a singular potential. So some new argument has to be found to treat the singular case.

\section{FUNCTIONAL SETTING AND PRELIMINARIES}

II.1. Functional framework. For any $\mu \leq 1 / 4$, we define

$$
\begin{aligned}
H_{0}^{1, \mu}(0,1):=\left\{z \in L^{2}(0,1) \cap H_{l o c}^{1}((0,1]) \mid\right. & z(0)=0=z(1) \\
& \left.\quad \text { and } \int_{0}^{1}\left(z_{x}^{2}-\frac{\mu}{x^{2}} z^{2}\right) d x<+\infty\right\} .
\end{aligned}
$$

In the case of a sub-critical parameter $\mu<1 / 4$, thanks to Hardy inequality (I. 2), it is easy to see that $H_{0}^{1, \mu}(0,1)=H_{0}^{1}(0,1)$. On the contrary, for the critical value $\mu=\mu_{\star}=1 / 4$, the space is enlarged (see [30] for a precise description of this space):

$$
H_{0}^{1}(0,1) \underset{\neq}{\subset} H_{0}^{1, \mu=1 / 4}(0,1)=: H^{\star}(0,1) .
$$

Next we prove

Lemma II.1. Let $\mu \leq 1 / 4$ be given. Then $H_{0}^{1, \mu}(0,1) \hookrightarrow L^{2}(0,1)$ with compact embedding.

Proof. For any $\mu<1 / 4, H_{0}^{1, \mu}(0,1)=H_{0}^{1}(0,1)$ so the result is well-known. Consider now $\mu=1 / 4$. Deriving some improved Hardy-Poincaré inequalities (see [30, Theorem 2.2]), Vázquez and Zuazua noticed that

$$
H^{\star}(0,1) \hookrightarrow W_{0}^{1, q}(0,1) \text { if } 1 \leq q<2 .
$$

Then, for $0 \leq s<1$, we use the fact that $W_{0}^{1, q}(0,1)$ is compactly embedded in $H_{0}^{s}(0,1)$ for suitable $q=q(s)$ close enough to 2 . It follows that

$$
H^{\star}(0,1) \hookrightarrow H_{0}^{s}(0,1) \text { with compact embedding if } 0 \leq s<1 \text {. }
$$

Finally, we conclude using the fact that $H_{0}^{s}(0,1)$ is compactly embedded in $L^{2}(0,1)$.

For any $\mu \leq 1 / 4$ and $z \in H_{0}^{1, \mu}(0,1)$, we consider the positive and negative parts of $z$ respectively defined by

$$
\begin{array}{ll}
z^{+}(x):=\max (z(x), 0), & x \in(0,1), \\
z^{-}(x):=-\min (0, z(x)), & x \in(0,1),
\end{array}
$$

so that $z=z^{+}-z^{-}$. We will need the following result of regularity for $z^{+}$and $z^{-}$: 
Lemma II.2. Let $\mu \leq 1 / 4$ and consider $z \in H_{0}^{1, \mu}(0,1)$. Then for any $1 \leq q<2$, $z^{+}$and $z^{-}$belong to $W^{1, q}(0,1)$. Moreover

$$
\left(z^{+}\right)_{x}=\left\{\begin{array}{ll|l}
z_{x} & \text { in }\{x \in(0,1) & z(x)>0\} \\
0 & \text { in }\{x \in(0,1) & z(x) \leq 0\}
\end{array}\right.
$$

and

$$
\left(z^{-}\right)_{x}=\left\{\begin{array}{ll|l}
0 & \text { in }\{x \in(0,1) & z(x) \geq 0\}, \\
-z_{x} & \text { in }\{x \in(0,1) & z(x)<0\} .
\end{array}\right.
$$

Proof. Consider $z \in H_{0}^{1, \mu}(0,1)$ with $\mu \leq 1 / 4$. From (II. 1) and (II. 2), we deduce that, for any $1 \leq q<2, z$ belongs to $W^{1, q}(0,1)$. Next, using Theorem V.1 in appendix, one deduce that $z^{+}, z^{-} \in W^{1, q}(0,1)$ and (II. 3) and (II. 4) hold true.

II.2. The unperturbed operator. Let us describe here the unperturbed operator corresponding to the heat equation with inverse square potential. We define it in the following way:

$$
\left\{\begin{array}{l}
D\left(A_{0}\right):=\left\{z \in H_{l o c}^{2}((0,1]) \cap H_{0}^{1, \mu}(0,1) \mid z_{x x}+\frac{\mu}{x^{2}} z \in L^{2}(0,1)\right\} \\
A_{0} z:=z_{x x}+\frac{\mu}{x^{2}} z
\end{array}\right.
$$

In this context, $A_{0}$ is a closed, self-adjoint, dissipative operator with dense domain in $L^{2}(0,1)$ (see [30]). Therefore $A_{0}$ is the infinitesimal generator of a $C_{0^{-}}$ semigroup of contractions in $L^{2}(0,1)$.

Moreover, from the spectral theory for self-adjoint operators with compact inverse (see [30]), we have:

Lemma II.3. Assume $\mu \leq 1 / 4$. There exists an nondecreasing sequence $\left(\bar{\lambda}_{k}\right)_{k \geq 1}$, $\bar{\lambda}_{k} \rightarrow+\infty$ as $k \rightarrow+\infty$, such that the eigenvalues of $A_{0}$ are given by $\left(-\bar{\lambda}_{k}\right)_{k \geq 1}$ and have finite multiplicity. Besides the corresponding eigenfunctions $\left\{\bar{\omega}_{k}\right\}_{k \geq 1}$ form a complete orthonormal system in $L^{2}(0,1)$.

Concerning, the eigenfunction associated to the first eigenvalue, we have the following result:

Lemma II.4. Assume $\mu \leq 1 / 4$. The first eigenvalue $-\bar{\lambda}_{1}$ is simple and the corresponding eigenfunction $\bar{\omega}_{1}$ satisfies

$$
\bar{\omega}_{1}(x)>0 \text { for all } x \in(0,1) \quad \text { or } \quad \bar{\omega}_{1}(x)<0 \text { for all } x \in(0,1) .
$$

Proof. The expression of the eigenfunctions of $A_{0}$ has been computed in [24] and we recall it in Proposition V.1 in appendix. So, using Proposition V.1, the first normalized eigenfunction is

$$
\bar{\omega}_{1}= \pm \frac{1}{\left|J_{\nu}^{\prime}\left(j_{\nu, 1}\right)\right|} \sqrt{x} J_{\nu}\left(j_{\nu, 1} x\right), \quad x \in(0,1),
$$

and since $j_{\nu, 1}$ is the first positive zero of $J_{\nu}$, this function does not vanish in $(0,1)$.

II.3. Perturbed operators. Next, for any $\alpha=\alpha(x) \in L^{\infty}(0,1)$ given, we consider now the perturbed operator

$$
A:=A_{0}+\alpha I \text { with domain } D(A):=D\left(A_{0}\right) .
$$

Then one can prove

Proposition II.1. Let $\mu \leq 1 / 4$ and $\alpha \in L^{\infty}(0,1)$ be given. Then the above operator $(A, D(A))$ satisfies

- $D(A)$ is compactly embedded and dense in $L^{2}(0,1)$. 
- $A: D(A) \rightarrow L^{2}(0,1)$ is the infinitesimal generator of a strongly continuous semi-group $e^{t A}$ of bouded linear operators on $L^{2}(0,1)$.

Problem (I. 4) can be rewritten in the Hilbert space $L^{2}(0,1)$ in the following way

$$
\left\{\begin{array}{l}
u^{\prime}(t)=A u(t), \quad t \in(0, T) \\
u(0)=u_{0}
\end{array}\right.
$$

In the following, we will simply denote by $\|\cdot\|$ the norm in $L^{2}(0,1)$ and by $\langle\cdot, \cdot\rangle$ the scalar product in $L^{2}(0,1)$. We recall (see [1]) that a weak solution of (II. 6) is a function $u \in \mathcal{C}^{0}\left([0, T] ; L^{2}(0,1)\right)$ such that, for every $v \in D\left(A^{\star}\right)$, the function $v \mapsto\langle u(t), v\rangle$ is absolutely continuous on $[0, T]$ and

$$
\frac{d}{d t}\langle u(t), v\rangle=\left\langle u(t), A^{\star} v\right\rangle, \quad \text { a.e. } t \in(0, T) .
$$

Theorem II.1. Let $\mu \leq 1 / 4$ be given. For every $\alpha \in L^{\infty}(0,1)$ and for every $u_{0} \in L^{2}(0,1)$, there exists a unique weak solution

$$
u \in \mathcal{B}(0, T):=\mathcal{C}^{0}\left([0, T] ; L^{2}(0,1)\right) \cap L^{2}\left(0, T ; H_{0}^{1, \mu}(0,1)\right)
$$

to (I. 4), which coincides with $e^{t A} u_{0}$.

Besides, once again from the spectral theory for self-adjoint operators with compact inverse, we have:

Lemma II.5. Assume $\mu \leq 1 / 4$ and $\alpha \in L^{\infty}(0,1)$. There exists an nondecreasing sequence $\left(\lambda_{k}\right)_{k \geq 1}, \lambda_{k} \rightarrow+\infty$ as $k \rightarrow+\infty$, such that the eigenvalues of $A$ are given by $\left(-\lambda_{k}\right)_{k \geq 1}$ and heve finite multiplicity. Besides the corresponding eigenfunctions $\left\{\omega_{k}\right\}_{k \geq 1}$ form a complete orthonormal system in $L^{2}(0,1)$. Moreover, the first eigenvalue of $A$ is given by:

$$
\lambda_{1}=\inf _{z \in D(A) \backslash\{0\}} \frac{-\langle A z, z\rangle}{\|z\|^{2}} .
$$

II.4. Maximum principle. For perturbed operators of the form $A=A_{0}+\alpha I$, we will also need the following result:

Lemma II.6. Let $\mu \geq 1 / 4$ be given. Let $T>0, \alpha \in L^{\infty}(0,1)$ and $u_{0} \in L^{2}(0,1)$ such that $u_{0} \geq 0$ in $(0,1)$. Consider $u \in \mathcal{B}(0, T)$ be the corresponding solution of (I. 4). Then $u \geq 0$ in $Q_{T}$.

Proof. Consider $u \in \mathcal{B}(0, T)$ the solution of (I. 4) and let us prove that $u^{-} \equiv 0$ in $Q_{T}$ (which suffices to show that $u \geq 0$ in $Q_{T}$ ). Multiplying the equation by $u^{-}$ and integrating on $(0,1)$, we get

$$
\int_{0}^{1}\left(u_{t} u^{-}-u_{x x} u^{-}-\frac{\mu}{x^{2}} u u^{-}-\alpha u u^{-}\right) d x=0 .
$$

Using $u=u^{+}-u^{-}$, we compute each term:

$$
\begin{gathered}
\int_{0}^{1} u_{t} u^{-} d x=\int_{0}^{1}\left(u^{+}-u^{-}\right)_{t} u^{-} d x=-\int_{0}^{1}\left(u^{-}\right)_{t} u^{-} d x=-\frac{1}{2} \frac{d}{d t} \int_{0}^{1}\left(u^{-}\right)^{2} d x \\
\int_{0}^{1} u_{x x} u^{-} d x=-\int_{0}^{1} u_{x}\left(u^{-}\right)_{x} d x=-\int_{0}^{1}\left(u^{+}-u^{-}\right)_{x}\left(u^{-}\right)_{x} d x=\int_{0}^{1}\left(\left(u^{-}\right)_{x}\right)^{2} d x \\
\int_{0}^{1} \frac{\mu}{x^{2}} u u^{-} d x=\int_{0}^{1} \frac{\mu}{x^{2}}\left(u^{+}-u^{-}\right) u^{-} d x=-\int_{0}^{1} \frac{\mu}{x^{2}}\left(u^{-}\right)^{2} d x \\
\int_{0}^{1} \alpha u u^{-} d x=\int_{0}^{1} \alpha\left(u^{+}-u^{-}\right) u^{-} d x=-\int_{0}^{1} \alpha\left(u^{-}\right)^{2} d x
\end{gathered}
$$


We deduce

$$
-\frac{1}{2} \frac{d}{d t} \int_{0}^{1}\left(u^{-}\right)^{2} d x-\int_{0}^{1}\left(\left(u^{-}\right)_{x}\right)^{2} d x+\int_{0}^{1} \frac{\mu}{x^{2}}\left(u^{-}\right)^{2} d x+\int_{0}^{1} \alpha\left(u^{-}\right)^{2} d x=0 .
$$

Hence

$$
\frac{d}{d t} \int_{0}^{1}\left(u^{-}\right)^{2} d x=2 \int_{0}^{1} \alpha\left(u^{-}\right)^{2} d x-2 \int_{0}^{1}\left[\left(\left(u^{-}\right)_{x}\right)^{2}-\frac{\mu}{x^{2}}\left(u^{-}\right)^{2}\right] d x .
$$

From Hardy inequality (I. 2 ) and the fact that $\mu \leq 1 / 4$, we have

so

$$
\int_{0}^{1} \frac{\mu}{x^{2}}\left(u^{-}\right)^{2} d x \leq \frac{1}{4} \int_{0}^{1} \frac{\left(u^{-}\right)^{2}}{x^{2}} d x \leq \int_{0}^{1}\left(\left(u^{-}\right)_{x}\right)^{2} d x
$$

Then we deduce

$$
-2 \int_{0}^{1}\left[\left(\left(u^{-}\right)_{x}\right)^{2}-\frac{\mu}{x^{2}}\left(u^{-}\right)^{2}\right] d x \leq 0
$$

$$
\frac{d}{d t} \int_{0}^{1}\left(u^{-}\right)^{2} d x \leq 2 \int_{0}^{1} \alpha\left(u^{-}\right)^{2} d x \leq 2\|\alpha\|_{L^{\infty}(0,1)} \int_{0}^{1}\left(u^{-}\right)^{2} d x .
$$

Using Gronwall's inequality, it follows that

$$
\forall t \in(0, T), \quad \int_{0}^{1} u^{-}(x, t)^{2} d x \leq \int_{0}^{1} u^{-}(x, 0)^{2} d x e^{2\|\alpha\|_{L^{\infty}(0,1)} t} .
$$

But $u_{0} \geq 0$ so $u^{-}(x, 0)=0$. This implies that $u^{-}(x, t) \equiv 0$ for $(x, t) \in Q_{T}$.

II.5. Specific perturbed operator. In this paragraph, we consider now some special pertubed operator that will be used later in order to exhibit a suitable bilinear control. We prove:

Lemma II.7. Let $u \in D\left(A_{0}\right)$ be given such that $u>0$ on $(0,1)$ and such that

Next consider the operator

$$
\frac{u_{x x}}{u}+\frac{\mu}{x^{2}} \in L^{\infty}(0,1)
$$

$$
A:=A_{0}+\alpha_{\star} I \text { with domain } D(A):=D\left(A_{0}\right),
$$

and where $\alpha_{\star}$ is defined by

$$
\alpha_{\star}(x):=-\frac{u_{x x}}{u}-\frac{\mu}{x^{2}} \text { for } x \in(0,1) .
$$

Let $\left(-\lambda_{k}\right)_{k \geq 1}$ and $\left\{\omega_{k}\right\}_{k \geq 1}$ be the eigenvalues and eigenfunctions of $A$ given in Lemma II. $\overline{5}$. Then the first eigenvalue $-\lambda_{1}$ is simple and its value is $\lambda_{1}=0$. Moreover, the corresponding normalized eigenfunction $\omega_{1}$ satisfies

$$
\left|\omega_{1}\right|=\frac{u}{\|u\|} .
$$

Besides, $\omega_{1}$ is the only element of $\left\{\omega_{k}\right\}_{k \geq 1}$ that does not change sign on $(0,1)$.

Proof. Let us compute

$$
A \frac{u}{\|u\|}=\frac{u_{x x}}{\|u\|}+\frac{\mu}{x^{2}} \frac{u}{\|u\|}+\alpha_{\star}(x) \frac{u}{\|u\|}=0 .
$$

It follows that $u /\|u\|$ is an eigenfunction (with norm 1) of $A$ associated to the eigenvalue $\lambda=0$. Hence there exists $k_{\star} \geq 1$ such that $\lambda_{k_{\star}}=0$ and

$$
\omega_{k_{\star}}=\frac{u}{\|u\|}>0 \text { or } \omega_{k_{\star}}=-\frac{u}{\|u\|}<0 .
$$

By orthogonality of the familly $\left\{\omega_{k}\right\}_{k \geq 1}$, we have

$$
\forall l \neq k_{\star}, \quad \int_{0}^{1} \omega_{k_{\star}}(x) \omega_{l}(x) d x=0 .
$$


Consequently, $\omega_{k_{\star}}$ is the only element of $\left\{\omega_{k}\right\}_{k \geq 1}$ that does not change sign in $(0,1)$.

Let us now prove that $k_{\star}=1$, that is $\lambda_{1}=0$. Since $-\lambda=0$ is an eigenvalue and since the sequence $\left(-\lambda_{k}\right)_{k \geq 1}$ is decreasing, we have $-\lambda_{1} \geq 0$ that is $\lambda_{1} \leq 0$. So it is sufficient to show that $\lambda_{1} \geq 0$.

We use the characterization of the first eigenvalue of $A$ :

$$
\lambda_{1}=\inf _{z \in D(A) \backslash\{0\}} \frac{-\langle A z, z\rangle}{\|z\|^{2}} .
$$

For any $z \in D(A)$, we compute

$$
\begin{aligned}
\langle A z, z\rangle & =\int_{0}^{1}\left(z_{x x}+\frac{\mu}{x^{2}} z+\alpha^{\star} z\right) z d x=\int_{0}^{1}\left(z_{x x} z+\frac{\mu}{x^{2}} z^{2}-\frac{u_{x x}}{u} z^{2}-\frac{\mu}{x^{2}} z^{2}\right) d x \\
& =\int_{0}^{1} z_{x x} z d x-\int_{0}^{1} u_{x x}\left(\frac{z^{2}}{u}\right) d x=-\int_{0}^{1} z_{x}^{2} d x+\int_{0}^{1} u_{x}\left(\frac{z^{2}}{u}\right) d x \\
& =-\int_{0}^{1} z_{x}^{2} d x+2 \int_{0}^{1}\left(\frac{u_{x}}{u} z\right) z_{x} d x-\int_{0}^{1} u_{x}^{2} \frac{z^{2}}{u^{2}} d x \\
& \leq-\int_{0}^{1} z_{x}^{2} d x+\int_{0}^{1}\left(\frac{u_{x}}{u} z\right)^{2} d x+\int_{0}^{1} z_{x}^{2} d x-\int_{0}^{1} u_{x}^{2} \frac{z^{2}}{u^{2}} d x=0 .
\end{aligned}
$$

It follows that $\langle A z, z\rangle \leq 0$ for any $z \in D(A)$ which implies that $\lambda_{1} \geq 0$.

It remains to prove that $\lambda_{1}$ is simple. Observe that

$$
\forall z \in D(A), \quad \frac{-\langle A z, z\rangle}{\|z\|^{2}}=Q(z)
$$

where $Q$ is the quadratic form defined by

$$
\forall z \in H_{0}^{1, \mu}(0,1), \quad Q(z):=\frac{1}{\|z\|^{2}} \int_{0}^{1}\left(z_{x}^{2}-\frac{\mu}{x^{2}} z^{2}-\alpha_{\star}(x) z^{2}\right) d x .
$$

Another characterization of the first eigenvalue $-A$ is

$$
\lambda_{1}=\inf _{z \in H_{0}^{1, \mu}(0,1) \backslash\{0\}} Q(z) .
$$

Any eigenfunction $\omega$ of $A$ associated to $\lambda_{1}$ is a minimizer of $Q$. Reciprocally, by standard arguments of the calculus of variations, any minimizer $\omega$ of $Q$ is an eigenfunction of $A$ corresponding to $\lambda_{1}$.

We argue by contradiction assuming that $\lambda_{2}=\lambda_{1}$ so that $\omega_{2}$ is another eigenfunction of $A$ associated to $\lambda_{1}$. It follows that $\omega_{2}$ is a minimizer of $Q$. By Lemma II.2, it is easy to show that $Q\left(\left|\omega_{2}\right|\right)=Q\left(\omega_{2}\right)$ so $\left|\omega_{2}\right|$ is also a minimizer of $Q$. Therefore $\left|\omega_{2}\right|$ is an eigenfunction associated to $\lambda_{1}$. So we get

$$
\left\{\begin{array}{l}
A\left|\omega_{2}\right|=\lambda_{1}\left|\omega_{2}\right|=0 \text { in }(0,1) \\
\left|\omega_{2}\right|(x=0)=0=\left|\omega_{2}\right|(x=1),
\end{array}\right.
$$

i.e.

We deduce

$$
\left\{\begin{array}{l}
-\left(\left|\omega_{2}\right|\right)_{x x}=\frac{\mu}{|x|^{2}}\left|\omega_{2}\right|+\alpha_{\star}(x)\left|\omega_{2}\right| \text { in }(0,1), \\
\left|\omega_{2}\right|(x=0)=0=\left|\omega_{2}\right|(x=1)
\end{array}\right.
$$

$\left\{\begin{array}{l}-\left(\left|\omega_{2}\right|\right)_{x x}+\left\|\alpha_{\star}\right\|_{L^{\infty}(0,1)}\left|\omega_{2}\right|=\frac{\mu}{|x|^{2}}\left|\omega_{2}\right|+\left(\left\|\alpha_{\star}\right\|_{L^{\infty}(0,1)}+\alpha_{\star}(x)\right)\left|\omega_{2}\right| \geq 0 \text { in }(0,1), \\ \left|\omega_{2}\right|(x=0)=0=\left|\omega_{2}\right|(x=1) .\end{array}\right.$

Since $\left|\omega_{2}\right| \not \equiv 0$, by strong maximum principle, we have $\left|\omega_{2}\right|>0$ in $(0,1)$. It follows that $\omega_{2}>0$ in $(0,1)$ or $\omega_{2}<0$ in $(0,1)$. This contredicts the fact that $\omega_{1}$ is the only element of $\left\{\omega_{k}\right\}_{k \geq 1}$ that does not change sign in $(0,1)$. 


\section{Proof of Theorem I.1}

In this section, we prove Theorem I.1. The proof is divided into the following 3 steps: first of all, we show that it is sufficient to consider some well-chosen subset of targets; secondly, for any $u_{d}$ in the previous subset, one exhibit some $\alpha_{\star}=\alpha_{\star}(x)$ such that $u_{d}$ is simply co-linear the first positive eigenfunction of the perturbed operator $A_{0}+\alpha_{\star} I$; finally, one construct a small perturbation $\alpha=\alpha_{\star}+\delta$ of $\alpha_{\star}$ that solves the question at hand.

III.1. Step 1. In a first step, we show that it is sufficient to prove the result for the following set of non-negative target states $u_{d}$ :

$$
u_{d} \in D\left(A_{0}\right), \quad u_{d}>0 \text { in }(0,1) \text { such that } \frac{u_{d, x x}}{u_{d}}+\frac{\mu}{x^{2}} \in L^{\infty}(0,1) .
$$

Indeed let us consider $u_{d}$ as in Theorem I.1, that is $u_{d}$ satisfying $u_{d} \in L^{2}(0,1)$ and $u_{d} \geq 0$ in $(0,1)$. Let us fix $\varepsilon>0$. Using a regularization by convolution, one can find a function $u_{d}^{\varepsilon}$ such that

$$
u_{d}^{\varepsilon} \in \mathcal{C}^{\infty}([0,1]), \quad u_{d}^{\varepsilon}>0 \text { in }(0,1) \text { such that }\left\|u_{d}-u_{d}^{\varepsilon}\right\| \leq \frac{\varepsilon}{2} .
$$

Let us denote $\bar{\omega}_{1}$ the first positive eigenfunction (corresponding to the eigenvalue $-\bar{\lambda}_{1}$ ) of $A_{0}$ with norm 1 that we introduced in Lemma II.4. Of course, $\bar{\omega}_{1}$ belongs to $D\left(A_{0}\right)$ and is a solution of the following Sturm-Liouville problem

$$
\left\{\begin{array}{l}
\bar{\omega}_{1, x x}+\frac{\mu}{x^{2}} \bar{\omega}_{1}+\bar{\lambda}_{1} \bar{\omega}_{1}=0, \quad x \in(0,1), \\
\bar{\omega}_{1}(0)=0=\bar{\omega}_{1}(1) .
\end{array}\right.
$$

Consider some cut-off function as follows : for $\sigma>0$ small, $\xi_{\sigma} \in \mathcal{C}^{\infty}([0,1])$ is such that

$$
\begin{cases}0 \leq \xi_{\sigma}(x) \leq 1, & x \in[0,1], \\ \xi_{\sigma}(x)=1, & x \in[0, \sigma / 2], \\ \xi_{\sigma}(x)=0, & x \in[\sigma, 1] .\end{cases}
$$

And define

$$
\bar{u}_{d}^{\varepsilon}(x):=\xi_{\sigma}(x) \bar{\omega}_{1}(x)+\left(1-\xi_{\sigma}(x)\right) u_{d}^{\varepsilon}(x) .
$$

Since $\bar{u}_{d}^{\varepsilon}=\bar{\omega}_{1}$ on $[0, \sigma / 2]$, one can easily check that $\bar{u}_{d}^{\varepsilon} \in D\left(A_{0}\right)$. Moreover $\bar{u}_{d}^{\varepsilon}>0$ using the fact that $u_{d}^{\varepsilon}>0$ and $\bar{\omega}_{1}>0$.

Finally, still using the fact that $\bar{u}_{d}^{\varepsilon}=\bar{\omega}_{1}$ on $[0, \sigma / 2]$, we observe that

$$
\left(\frac{\bar{u}_{d, x x}^{\varepsilon}}{\bar{u}_{d}^{\varepsilon}}+\frac{\mu}{x^{2}}\right)_{\mid[0, \sigma / 2]}=\left(\frac{\bar{\omega}_{1, x x}}{\bar{\omega}_{1}}+\frac{\mu}{x^{2}}\right)_{\mid[0, \sigma / 2]}=-\bar{\lambda}_{1} \in L^{\infty}(0, \sigma / 2) .
$$

And we can deduce that

$$
\frac{\bar{u}_{d, x x}^{\varepsilon}}{\bar{u}_{d}^{\varepsilon}}+\frac{\mu}{x^{2}} \in L^{\infty}(0,1) .
$$

So $\bar{u}_{d}^{\varepsilon}$ belongs to the set described in (III. 1).

Now let us show that it is sufficient to steer the solution near $\bar{u}_{d}^{\varepsilon}$ instead of $u_{d}$ : we first estimate

$$
\left\|u_{d}^{\varepsilon}-\bar{u}_{d}^{\varepsilon}\right\|^{2}=\int_{0}^{1} \xi_{\sigma}(x)^{2}\left(\bar{\omega}_{1}(x)-u_{d}^{\varepsilon}(x)\right)^{2} d x \leq \int_{0}^{\sigma}\left(\bar{\omega}_{1}(x)-u_{d}^{\varepsilon}(x)\right)^{2} d x .
$$

Therefore it is possible to choose $\sigma>0$ small enough so that

Finally we obtain

$$
\left\|u_{d}^{\varepsilon}-\bar{u}_{d}^{\varepsilon}\right\|^{2} \leq \frac{\varepsilon^{2}}{4}
$$

$$
\left\|u_{d}-\bar{u}_{d}^{\varepsilon}\right\| \leq\left\|u_{d}-u_{d}^{\varepsilon}\right\|+\left\|u_{d}^{\varepsilon}-\bar{u}_{d}^{\varepsilon}\right\| \leq \frac{\varepsilon}{2}+\frac{\varepsilon}{2}=\varepsilon .
$$


III.2. Step 2. In this second step, for any $u_{d}$ such that (III. 1) holds, we select some $\alpha_{\star}=\alpha_{\star}(x)$ such that $u_{d}$ becomes co-linear the first positive eigenfunction of the perturbed operator $A_{0}+\alpha_{\star} I$.

Indeed, let us now consider $u_{0}$ non-zero and non-negative in $L^{2}(0,1)$ and $u_{d}$ as in (III. 1). And define

$$
\alpha_{\star}(x):=-\frac{u_{d, x x}}{u_{d}}-\frac{\mu}{x^{2}}, \quad x \in(0,1) .
$$

Since $\alpha_{\star} \in L^{\infty}(0,1)$, we can define the perturbed operator $A:=A_{0}+\alpha_{\star} I$ with domain $D(A):=D\left(A_{0}\right)$. As in Lemma II.5, we denote by $\left(-\lambda_{k}\right)_{k \geq 1}$ and $\left\{\omega_{k}\right\}_{k>1}$ the eigenvalues and the corresponding orthonormal eigenfunctions of $A$. Here, for $\omega_{1}$, we choose the positive eigenfunction associated to the first eigenvalue. Then, applying Lemma II.7, we have

$$
\lambda_{1}=0 \quad \text { and } \quad \omega_{1}(x)=\frac{u_{d}(x)}{\left\|u_{d}\right\|}>0, \quad x \in(0,1),
$$

so $u_{d}$ and $\omega_{1}$ are co-linear.

III.3. Step 3. In this last step, we are now ready to choose the (static) bilinear control that allows us to achieve our goal. It is constructed in the following way as a small perturbation of $\alpha_{\star}$ : we set

$$
\alpha(x):=\alpha_{\star}(x)+\delta
$$

where $\delta \in \mathbb{R}$ will be chosen later.

Observe that, adding $\delta$ to $\alpha_{\star}$ generates a shift on the eigenvalues corresponding to $\alpha_{\star}$ (they change from $\left(-\lambda_{k}\right)_{k \geq 1}$ to $\left.\left(-\lambda_{k}+\delta\right)_{k \geq 1}\right)$ whereas the eigenfunctions $\left\{\omega_{k}\right\}_{k \geq 1}$ remain the same.

The solution of (I. 4) corresponding to the choice of $\alpha$ given in (III. 7) can be written in Fourier series representation as

$$
u(x, t)=\sum_{k=1}^{+\infty} e^{\left(-\lambda_{k}+\delta\right) t}\left\langle u_{0}, \omega_{k}\right\rangle \omega_{k}(x)=e^{\delta t}\left\langle u_{0}, \omega_{1}\right\rangle \omega_{1}(x)+r(x, t)
$$

where

$$
r(x, t):=\sum_{k=2}^{+\infty} e^{\left(-\lambda_{k}+\delta\right) t}\left\langle u_{0}, \omega_{k}\right\rangle \omega_{k}(x) .
$$

Since $u_{d}=\left\|u_{d}\right\| \omega_{1}$, we obtain

$$
\begin{aligned}
\left\|u(\cdot, t)-u_{d}\right\| & \leq\left\|e^{\delta t}\left\langle u_{0}, \omega_{1}\right\rangle \omega_{1}(x)-\right\| u_{d}\left\|\omega_{1}\right\|+\|r(x, t)\| \\
& =\left|e^{\delta t}\left\langle u_{0}, \omega_{1}\right\rangle-\left\|u_{d}\right\|\right|+\|r(x, t)\| .
\end{aligned}
$$

Next we recall that $-\lambda_{k} \leq-\lambda_{2}$ for all $k \geq 2$. So

$\|r(x, t)\|^{2}=\sum_{k=2}^{+\infty} e^{2\left(-\lambda_{k}+\delta\right) t}\left|\left\langle u_{0}, \omega_{k}\right\rangle\right|^{2} \leq e^{2\left(-\lambda_{2}+\delta\right) t} \sum_{k=2}^{+\infty}\left|\left\langle u_{0}, \omega_{k}\right\rangle\right|^{2} \leq e^{2\left(-\lambda_{2}+\delta\right) t}\left\|u_{0}\right\|^{2}$.

For $\varepsilon>0$ fixed, let us choose $T_{\varepsilon}>0$ such that

$$
e^{-\lambda_{2} T_{\varepsilon}}=\varepsilon \frac{\left\langle u_{0}, u_{d}\right\rangle}{\left\|u_{0}\right\|\left\|u_{d}\right\|^{2}} \quad \text { i.e. } T_{\varepsilon}=\frac{-1}{\lambda_{2}} \ln \left(\varepsilon \frac{\left\langle u_{0}, u_{d}\right\rangle}{\left\|u_{0}\right\|\left\|u_{d}\right\|^{2}}\right)
$$

which is possible since $\lambda_{1}=0$ is simple so $\lambda_{2} \neq 0$. Since $u_{0} \in L^{2}(0,1), u_{0} \geq 0$, $u_{0} \neq 0$ and $\omega_{1}>0$ (see (III. 6)), we get

$$
\left\langle u_{0}, \omega_{1}\right\rangle=\int_{0}^{1} u_{0}(x) \omega_{1}(x) d x>0 .
$$


It is then possible to choose $\delta_{\varepsilon}$ such that

that is

$$
e^{\delta_{\varepsilon} T_{\varepsilon}}=\frac{\left\|u_{d}\right\|}{\left\langle u_{0}, \omega_{1}\right\rangle}=\frac{\left\|u_{d}\right\|^{2}}{\left\langle u_{0}, u_{d}\right\rangle}
$$

$$
\delta_{\varepsilon}=\frac{1}{T_{\varepsilon}} \ln \left(\frac{\left\|u_{d}\right\|^{2}}{\left\langle u_{0}, u_{d}\right\rangle}\right) .
$$

We conclude that, for $\alpha(x)=\alpha_{\star}(x)+\delta_{\varepsilon}$,

$$
\left\|u\left(\cdot, T_{\varepsilon}\right)-u_{d}\right\| \leq e^{\left(-\lambda_{2}+\delta_{\varepsilon}\right) T_{\varepsilon}}\left\|u_{0}\right\|=e^{-\lambda_{2} T_{\varepsilon}} \frac{\left\|u_{d}\right\|^{2}}{\left\langle u_{0}, u_{d}\right\rangle}\left\|u_{0}\right\|=\varepsilon .
$$

So we proved that system (I. 4) is non-negatively globally approximately controllable in $L^{2}(0,1)$ by means of static controls $\alpha=\alpha(x)$ in $L^{\infty}(0,1)$. Moreover, by the maximum principle stated in Lemma II.6, the corresponding solution to (I. 4) remains non-negative at all times.

\section{Proof of Theorems I.2 AND I.3}

IV.1. Proof of Theorem I.2. The result directly follows from the proof of Theorem I.1. It is sufficient to observe that inequlity (III. 8) of step 3 still holds true under the assumptions of Theorem I.2. Indeed

$$
\left\langle u_{0}, \omega_{1}\right\rangle=\int_{0}^{1} u_{0}(x) \omega_{1}(x) d x=\int_{0}^{1} u_{0}(x) \frac{u_{d}(x)}{\left\|u_{d}\right\|} d x=\frac{1}{\left\|u_{d}\right\|}\left\langle u_{0}, u_{d}\right\rangle>0 .
$$

IV.2. Proof of Theorem I.3. The proof of Theorem I.3 follows the lines of the proof of Theorem I.1. So let us first establish the corresponding preliminar results.

Consider $V(x)$ satisfying (I. 5). We introduce the associated functional space:

$$
\begin{aligned}
H_{0}^{1, V}(0,1):=\left\{z \in L^{2}(0,1) \cap H_{l o c}^{1}((0,1])\right. & z(0)=0=z(1) \\
& \text { and } \left.\int_{0}^{1}\left(z_{x}^{2}-V(x) z^{2}\right) d x<+\infty\right\} .
\end{aligned}
$$

As for the inverse-square potential, thanks to Hardy inequality (I. 2), it is easy to see that $H_{0}^{1, V}(0,1)=H_{0}^{1}(0,1)$ when $\mu<1 / 4 . H_{0}^{1, V}(0,1)$ defines a new functional space only in the critical case $\mu=\mu_{\star}=1 / 4$.

As for Lemma II.1, using the improved Hardy-Poincaré inequalities in [30, Theorem 2.2], we can prove that

Lemma IV.1. Let $V(x)$ be given such that (I. 5) holds. Then $H_{0}^{1, V}(0,1) \hookrightarrow$ $L^{2}(0,1)$ with compact embedding.

With the same argument of proof, one can also show that Lemma II.2 is still true for any $z \in H_{0}^{1, V}(0,1)$.

Next we define the unperturbed operator:

$$
\left\{\begin{array}{l}
D\left(A_{0}\right):=\left\{z \in H_{l o c}^{2}((0,1]) \cap H_{0}^{1, V}(0,1) \mid z_{x x}+V(x) z \in L^{2}(0,1)\right\} \\
A_{0} z:=z_{x x}+V(x) z
\end{array}\right.
$$

From [30], we know that $A_{0}$ is a closed, self-adjoint, dissipative operator with dense domain in $L^{2}(0,1)$ and that Lemma II.3 still holds true.

Concerning, the eigenfunction associated to the first eigenvalue, we prove:

Lemma IV.2. Consider $V(x)$ satisfying (I. 5). The eigenfunction $\bar{\omega}_{1}$ corresponding to the first eigenvalue $-\bar{\lambda}_{1}$ of the above operator $A_{0}$ satisfies

$$
\bar{\omega}_{1}(x)>0 \text { for all } x \in(0,1) \quad \text { or } \quad \bar{\omega}_{1}(x)<0 \text { for all } x \in(0,1) \text {. }
$$


Proof. The proof of Lemma II.4 was based on an explicit expression of the eigenfunctions of $A_{0}$ in terms of Bessel functions obtained in [24]. Here we quote a result from Davila-Dupaigne [11]:

Proposition IV.1. Let $\Omega \subset R^{n}$ be a bounded smooth domain and consider $a \in$ $L_{\text {loc }}^{1}(\Omega), a \geq 0$. Assume that there exists $r>2$ such that

$$
\gamma(a):=\inf _{\varphi \in \mathcal{C}_{c}^{1}(\Omega)} \frac{\int_{\Omega}\left(|\nabla \varphi|^{2}-a(x) \varphi^{2}\right)}{\left(\int_{\Omega}|\varphi|^{r}\right)^{2 / r}}>0 .
$$

Define $H$ as the completion of $\mathcal{C}_{c}^{\infty}(\Omega)$ with respect to the norm

$$
\|\varphi\|_{H}^{2}:=\int_{\Omega}\left(|\nabla \varphi|^{2}-a(x) \varphi^{2}\right) \text {. }
$$

Then the operator $L:=-\Delta-a(x)$ with domain $D(L):=\{u \in H \quad \mid \Delta u-a(x) u \in$ $H\}$ has a positive first eigenvalue

$$
\bar{\lambda}_{1}=\inf _{\varphi \in H \backslash\{0\}} \frac{\int_{\Omega}\left(|\nabla \varphi|^{2}-a(x) \varphi^{2}\right)}{\int_{\Omega} \varphi^{2}},
$$

which is simple. The above quotient is attained at a positive $\bar{\varphi}_{1} \in H$ that satisfies

$$
\begin{cases}-\Delta \bar{\varphi}_{1}-a(x) \bar{\varphi}_{1}=\bar{\lambda}_{1} \bar{\varphi}_{1}, & \text { in } \Omega, \\ \bar{\varphi}_{1}=0, & \text { on } \partial \Omega .\end{cases}
$$

We see that the result simply follows from Proposition IV.1 applied with $n=1$, $\Omega=(0,1)$ and $a(x)=V(x)$. So it suffices to prove that $a(x)=V(x)$ satifies assumption (IV. 2).

We recall the following improved Hardy-Poincaré inequality from [30]: for all $1 \leq q<2$, there exists $C_{q}>0$ such that, for all $\varphi \in H_{0}^{1}(0,1)$,

$$
\int_{0}^{1}\left(|\nabla \varphi|^{2}-\frac{\mu_{\star}}{x^{2}} \varphi^{2}\right) \geq C_{q}\|\varphi\|_{W_{0}^{1, q}(0,1)}^{2} .
$$

Since $a=V$ satisfies assumption (I. 5), we deduce that, for all $1 \leq q<2$ and for all $\varphi \in \mathcal{C}_{c}^{1}(\Omega)$,

$$
\int_{0}^{1}\left(|\nabla \varphi|^{2}-a(x) \varphi^{2}\right) \geq C_{q}\|\varphi\|_{W_{0}^{1, q}(0,1)}^{2} .
$$

Next we use classical Sobolev embeddings. For $\Omega$ bounded domain of $\mathbb{R}^{n}$ with Lipschitz boundary, we have : for all $q$ such that $n<q<\infty$,

$$
W^{1, q}(\Omega) \hookrightarrow \mathcal{C}^{0,1-n / q}(\Omega) .
$$

Let us now choose (for example) $q=3 / 2$ so that $1 \leq q<2$ and $1=n<q<\infty$ and apply this to $\Omega=(0,1)$. It follows in particular that

$$
W^{1, q}(0,1) \hookrightarrow \mathcal{C}^{0}(0,1) .
$$

So there exists $c>0$ such that

$$
\sup _{x \in[0,1]}|\varphi(x)| \leq c\|\varphi\|_{W_{0}^{1, q}(0,1)} .
$$

Finally, fix $r>2$. Then

$$
\left(\int_{\Omega}|\varphi|^{r}\right)^{2 / r} \leq \sup _{x \in[0,1]}|\varphi(x)| \leq c\|\varphi\|_{W_{0}^{1, q}(0,1)} \leq \frac{c}{\sqrt{C_{q}}} \int_{0}^{1}\left(|\nabla \varphi|^{2}-a(x) \varphi^{2}\right)
$$

and (IV. 2) follows.

Next, for any $\alpha \in L^{\infty}\left(Q_{T}\right)$ given, we consider the perturbed operator

$$
A:=A_{0}+\alpha I \text { with domain } D(A):=D\left(A_{0}\right) \text {. }
$$


One can easily see that, under assumption (I. 5), Proposition II.1 together with the well-posedness Theorem II.1 (replacing the space $H_{0}^{1, \mu}(0,1)$ by $H_{0}^{1, V}(0,1)$ ) and the spectral Lemma II.5 are still true.

With similar proofs, one can see that the maximum principle for perturbed operators stated in Lemma II.6 holds unchanged for the solutions of (I. 6) whereas Lemma II. 7 is simply replaced by

Lemma IV.3. Assume $V(x)$ is given such that (I. 5) holds. Let $u \in D\left(A_{0}\right)$ be given such that $u>0$ on $(0,1)$ and such that

$$
\frac{u_{x x}}{u}+V(x) \in L^{\infty}(0,1) .
$$

Next consider the operator

$$
A:=A_{0}+\alpha_{\star} I \text { with domain } D(A):=D\left(A_{0}\right),
$$

and where $\alpha_{\star}$ is defined by

$$
\alpha_{\star}(x):=-\frac{u_{x x}}{u}-V(x) \text { for } x \in(0,1) .
$$

Let $\left(-\lambda_{k}\right)_{k>1}$ and $\left\{\omega_{k}\right\}_{k>1}$ be the eigenvalues and eigenfunctions of $A$ given in Lemma II.5. Then $\lambda_{1}=0$ is simple and

$$
\lambda_{1}=0 \quad \text { and } \quad\left|\omega_{1}\right|=\frac{u}{\|u\|} .
$$

Moreover, $u /\|u\|$ and $-u /\|u\|$ are the only eigenfunctions of $A$ with norm 1 that do not change sign on $(0,1)$.

This concludes the generalization of all preliminaries results. Finally, it is easy to see that the proof of Theorem I.1 can now be rewritten replacing $\mu / x^{2}$ by $V(x)$ and leads to Theorem I.3.

\section{APPENDIX}

This section is devoted to the statements of various technical results from literature that we use throughout this paper.

Let us first of all recall the following result from [23, Appendix A] that concerns the regularity of the negative and positive parts of a function:

Theorem V.1. Let $\Omega \subset \mathbb{R}^{n}$ and $1 \leq s \leq \infty$. Consider $v: \Omega \rightarrow \mathbb{R}$ such that $v \in W^{1, s}(\Omega)$. Then $v^{+}, v^{-} \in W^{1, s}(\Omega)$ and, for all $1 \leq i \leq n$,

and

$$
\left(v^{+}\right)_{x_{i}}=\left\{\begin{array}{ll|l}
v_{x_{i}} & \text { in }\{x \in \Omega & v(x)>0\} \\
0 & \text { in }\{x \in \Omega & v(x) \leq 0\}
\end{array}\right.
$$

$$
\left(v^{-}\right)_{x_{i}}=\left\{\begin{array}{ll|l}
0 & \text { in }\{x \in \Omega \mid & v(x) \geq 0\}, \\
-v_{x_{i}} & \text { in }\{x \in \Omega \mid & v(x)<0\} .
\end{array}\right.
$$

Next we recall the following expression of the eigenfunctions of $A_{0}$ (defined in (II. 5)) that have been computed in [24]:

Proposition V.1. Assume that $\mu$ is given such that $\mu \leq 1 / 4$ and define

$$
\nu:=\sqrt{\frac{1}{4}-\mu}
$$

We denote by $J_{\nu}$ the Bessel function of first kind of order $\nu$ and we denote $0<$ $j_{\nu, 1}<j_{\nu, 2}<\cdots<j_{\nu, n}<\cdots \rightarrow+\infty$ as $n \rightarrow+\infty$ the sequence of positive zeros of $J_{\nu}$. Then the admissible eigenvalues $\left(-\bar{\lambda}_{n}\right)_{n \geq 1}$ of $A_{0}$ are determined by

$$
\forall n \geq 1, \quad \bar{\lambda}_{n}=\left(j_{\nu, n}\right)^{2}
$$


and corresponding (normalized) eigenfunctions are given by

$$
\forall n \geq 1, \quad \Phi_{n}(x)=\frac{1}{\left|J_{\nu}^{\prime}\left(j_{\nu, n}\right)\right|} \sqrt{x} J_{\nu}\left(j_{\nu, n} x\right), \quad x \in(0,1) .
$$

\section{REFERENCES}

[1] J.M. Ball, Strongly continuous semigroups, weak solutions, and the variation of constants formula, Proc. Amer. Math. Soc. 63 (1977), 370-373.

2] P. Baras, J. Goldstein, Remarks on the inverse square potential in quantum mechanics, Differential equations (Birmingham, Ala., 1983), 31-35, North-Holland Math. Stud., 92, NorthHolland, Amsterdam, 1984.

[3] P. Baras, J. Goldstein, The heat equation with a singular potential, Trans. Amer. Math. Soc. 284 (1984), no. 1, 121-139.

[4] U. Biccari, E. Zuazua, Null controllability for a heat equation with a singular inverse-square potential involving the distance to the boundary function, J. Differential Equations 261 (2016), no. 5, 2809-2853.

[5] P. Cannarsa, G. Floridia, Approximate controllability for linear degenerate parabolic problems with bilinear control, Proc. Evolution equations and Materials with Memory 2010, Casa Editrice Università La Sapienza Roma (2011) 19-39.

[6] P. Cannarsa, G. Floridia, Approximate multiplicative controllability for degenerate parabolic problems with Robin boundary conditions, Communications in Applied and Industrial Mathematics, vol. 2, no. 2 (2011),

[7] P. Cannarsa, G. Floridia, A. Y. Khapalov, Multiplicative controllability for semilinear reaction-diffusion equations with finitely many changes of sign, J. Math. Pures Appl. (9) 108 (2017), no. 4, 425-458.

[8] P. Cannarsa, A. Y. Khapalov, Multiplicative controllability for reaction-diffusion equations with target states admitting finitely many changes of sign, Discrete Contin. Dyn. Syst. Ser. B 14 (2010), no. 4, 1293-1311.

[9] P. Cannarsa, P. Martinez, J. Vancostenoble, Carleman estimates for a class of degenerate parabolic operators, SIAM J. Control Optim. 47, (2008), no. 1, 1-19.

[10] C. Cazacu, Controllability of the heat equation with an inverse-square potential localized on the boundary, SIAM Journal of Control and Optimization 52 (4), 2055-2089.

[11] J. Davila, L. Dupaigne, Comparison results for PDEs with a singular potential, Proceedings of the Royal Society of Edinburgh Section A Mathematics 133(01), 61-83. February 2003.

[12] S. Ervedoza, Control and stabilization properties for a singular heat equation with an inversesquare potential, Comm. Partial Differential Equations 33 (2008), no. 10-12, 1996-2019.

[13] E. Fernandez-Cara, E. Zuazua, The cost of approximate controllability for heat equations: the linear case, Adv. Differential equations 5 (2000), No 4-6, 465-514.

[14] G. Floridia, Approximate controllability for nonlinear degenerate parabolic problems with bilinear control, Journal of Differential Equations Volume 257, Issue 9, 1 November 2014, 3382-3422.

[15] G. Floridia, Nonnegative controllability for a class of nonlinear degenerate parabolic equations, preprint.

[16] M. Fotouhi, L. Salimi, Null controllability of degenerate/singular parabolic equations, Journal of Dynamical and Control Systems, October 2012, Volume 18, Issue 4, pp 573-602.

[17] A. Hajjaj, L. Maniar, J. Salhi, Carleman estimates and null controllability of degenerate/singular parabolic systems, Electronic Journal of Di erential Equations, Vol. 2016 (2016), No. 292, pp. 1-25.

[18] G. H. Hardy, J. E. Littlewood, G. Pólya, "Inequalities", 2d ed. Cambridge, at the University Press, 1952

[19] A. Khapalov, Bilinear control for global controllability of the semilinear parabolic equations with superlinear terms. Control of nonlinear distributed parameter systems, (College Station, TX, 1999), 139-155, Lecture Notes in Pure and Appl. Math., 218, Dekker, New York, 2001.

[20] A. Khapalov, Global non-negative controllability of the semilinear parabolic equation governed by bilinear control, ESAIM Control Optim. Calc. Var. 7 (2002), 269-283.

[21] A.Y. Khapalov, Controllability of the semilinear parabolic equation governed by a multiplicative control in the reaction term: a qualitative approach, SIAM J. Control Optim. 41 (2003), no. 6, 18861900.

[22] A. Y. Khapalov, Controllability of partial differential equations governed by multiplicative controls, Lecture Notes in Mathematics, 1995. Springer-Verlag, Berlin, 2010. xvi+284 pp. ISBN: 978-3-642-12412-9 
[23] D. Kinderlehrer, G. Stampacchia, An introduction to variational inequalities and their applications, Classics Appl. Math, SIAM, 2000.

[24] P. Martinez, J.Vancostenoble, The cost of boundary controllability for a parabolic equation with inverse square potential, submitted.

[25] B. Opic, A. Kufner, Hardy-type Inequalities, Pitman Research Notes in Math., Vol. 219, Longman, 1990.

[26] M. Ouzahra, Approximate and exact controllability of a reaction-diffusion equation governed by bilinear control, European Journal of Control 32 (2016), 32-38.

[27] J. Vancostenoble, Improved Hardy-Poincaré inequalities and sharp Carleman estimates for degenerate/singular parabolic problems, Discrete and Continuous Dynamical Systems - Series S, American Institute of Mathematical Sciences, 2011, 4 (3), p. 761-790.

[28] J. Vancostenoble, Lipschitz stability in inverse source problems for singular parabolic equations, Communications in Partial Differential Equations, 2011, 36 (8), p. 1287-1317.

[29] J. Vancostenoble, E. Zuazua, Null controllability for the heat equation with singular inversesquare potentials, J. Funct. Anal. 254 (2008), no. 7, 1864-1902.

[30] J. L. Vázquez, E. Zuazua, The Hardy inequality and the asymptotic behaviour of the heat equation with an inverse-square potential, J. Funct. Anal. 173 (2000), no. 1, 103-153.

Institut de Mathématiques de Toulouse, UMR CNRS 5219, Université Paul Sabatier Toulouse III, 118 route de Narbonne, 31062 Toulouse Cedex 4, France

E-mail address: vancoste@math.univ-toulouse.fr 